\title{
Two-center prospective randomized controlled trial of Blake versus Portex drains after cardiac surgery
}

Neil Roberts, MRCS, ${ }^{a}$ Maria Boehm, RGN, ${ }^{\text {b }}$ Madeline Bates, MB, ${ }^{a}$ Peter C. Braidley, FRCS, ${ }^{a}$ Graham J. Cooper, FRCS, and Tom J. Spyt, FRCS ${ }^{b}$

From the Department of Cardiac Surgery, Northern General Hospital, ${ }^{\text {a }}$ Sheffield, United Kingdom, and the Department of Cardiac Surgery, Glenfield Hospital, ${ }^{\text {b }}$ Leicester, United Kingdom.

This study was supported by an educational grant from Ethicon, Inc, the manufacturer of the Blake drain.

Received for publication Feb 23, 2006; revisions received June 7, 2006; accepted for publication June 15, 2006.

Address for reprints: Mr Neil Roberts, Research Registrar Cardiac Surgery, St Georges Hospital, Blackshaw Rd, London SW17 0QT, United Kingdom (E-mail: neil.roberts@ stgeorges.nhs.uk).

J Thorac Cardiovasc Surg 2006;132:1042-6 $0022-5223 / \$ 32.00$

Copyright () 2006 by The American Association for Thoracic Surgery

doi:10.1016/j.jtcvs.2006.06.031
Objective: To compare the efficacy of two drain types after cardiac surgery in a randomized controlled trial, with primary outcome measure being depth of pericardial effusion 3 to 5 days after drain removal.

Methods: A prospective randomized controlled trial was conducted at two university hospitals. A total of 199 patients undergoing first-time cardiac procedures were randomized at surgery to receive either small-bore flexible Blake drains (19F) or larger Portex drains (28F). Drains were removed according to the study protocol and patients underwent echocardiography 3 to 5 days after drain removal to measure residual pericardial effusion.

Results: Ninety patients received Blake drains and 109 patients received Portex drains. There were no statistically significant differences in preoperative variables between the groups. There was no difference in the number of drains inserted per patient between groups (Blake $2.1 \pm 0.4$ vs Portex $2.0 \pm 0.5$ ). Mean difference in size of pericardial effusion between groups was $1.96 \mathrm{~mm}$ (95\% confidence interval $-0.02,3.95 \mathrm{~mm}$ ), which did not exceed the predefined non-inferiority margin of $10 \mathrm{~mm}$. There was no significant difference in the mean maximal hourly drainage rate between groups (Blake $94.7 \mathrm{~mL}$ vs Portex $123.1 \mathrm{~mL} ; P=.070$ ) or in the total drainage rates (Blake $541 \mathrm{~mL}$ vs Portex $679 \mathrm{~mL} ; P=.066$ ). Although the Blake group had a higher percentage of patients with detectable effusion (46.3\% vs $27.4 \%$; $P=.011)$ than the Portex group, there was no difference in need for late drainage of pericardial effusions (1.1\% vs $1.9 \%)$ or insertion of further chest drains ( $8.8 \%$ vs $7.2 \%)$.

Conclusion: The performance of small-bore Blake drains is not inferior to that of standard Portex drains after cardiac surgery.

$\mathrm{C}$ ardiac surgery requires the placement of drains in the mediastinum and sometimes in pleural cavities at the end of the surgical procedure. This is to ensure adequate drainage of blood to prevent tamponade and also to detect hemorrhage.

The usual method of drainage is to place large-bore (28F-36F) rigid Portex tubes (Portex, Inc, Hythe, Kent, United Kingdom) in the chest before wound closure. These drains are effective but cause pain, ${ }^{1}$ may interfere with bypass grafts, ${ }^{2,3}$ and may provoke cardiac arrhythmias. Despite encouraging reports of the use of smaller silicone rubber Blake drains ${ }^{4}$ (Ethicon, Inc, Somerville, NJ) and then convincing retrospective study data, ${ }^{5}$ the routine use of such drains after cardiac surgery has not been generally accepted.

Blake drains are small (10-19F) and have a fluted design, which allows a large surface area for drainage despite the small size. The drainage channels running along the side of the Blake drains reduce the likelihood of tissue invagination into the drains and hence may be less likely to interfere with coronary grafts. 


\section{Abbreviations and Acronyms \\ CIA $=$ Confidence Interval Analysis \\ SAS $=$ Statistical Analysis Software}

A prospective randomized trial confirmed that Blake drains are less painful than traditional large-bore drains, ${ }^{6}$ but no efficacy data are available based on a larger prospective randomized controlled trial.

Our study was designed to investigate the efficacy of the two drain types in a randomized controlled trial with primary outcome measure being depth of pericardial effusion postoperatively.

\section{Methods}

\section{Patient Population and Clinical Management}

Consecutive patients undergoing elective first-time cardiac surgery were invited to participate in the study subject to the exclusion criteria described below. Patient recruitment took place between September 2003 and September 2004 with a total of 199 patients treated.

Local research ethics committees from the two participating centers approved the study and written consent was obtained from all patients.

Patients undergoing second-time procedures, emergency or inhouse urgent surgery, triple valve surgery, concomitant carotid endarterectomy, and those with preoperative creatinine values greater than $180 \mathrm{mmol} / \mathrm{L}$ were excluded.

Anticoagulants were discontinued 5 days before the operation. Antiplatelet agents were administered 6 hours after the operation unless there was significant postoperative bleeding. Antiplatelet agents were then commenced on day 1 after the operation. Recipients of mechanical valves received warfarin on postoperative day 1. Recipients of tissue valves received high-dose aspirin or warfarin depending on individual consultant protocol.

Patients were randomly assigned to receive either Blake (19F) or Portex $(28 \mathrm{~F})$ drains in the theater, immediately before drain insertion. The protocol of the study did not specify the site of drain placement; individual consultants followed their standard surgical practice, and insertion sites, number, and location of drains were recorded. All consultants in the study inserted drains via the mediastinum; there were no insertions via the lateral chest wall.

All drains were connected to a chest drain bottle with an underwater seal and were subject to 2.5 - to $5-\mathrm{kPa}$ suction from the time of chest closure.

Drains were removed the day after the operation if no air leak was present and if total drainage was less than $20 \mathrm{~mL} / \mathrm{h}$ for at least 2 hours.

\section{Echocardiographic Assessment}

Transthoracic echocardiography was carried out between days 3 and 5 after drain removal to evaluate the presence of pericardial effusion. Echocardiography was performed by dedicated sonographers in each center who were blinded to drain type used. The echocardiographer used a standard form that detailed eight sites for measurements of pericardial effusion depth. At each site three separate measurements were made, which were then averaged. The largest of these averages was recorded and used for analysis.

All chest x-ray films were reviewed by a radiologist in accordance with standard hospital practice, and the presence of pneumothoraces and pleural effusions was reported. Decisions to insert further chest drains or to drain pericardial effusions were made depending on the need of the individual patient concerned.

Adverse events were recorded and monitored throughout the duration of the trial, with regular communication between the two centers.

\section{Statistical Analysis}

Statistical analysis was conducted with SAS (Statistical Analysis Software) version 8.02 software (SAS Institute, Inc, Cary, NC). Normally distributed continuous variables were analyzed by a Student $t$ test. Nonnormally distributed continuous data were analyzed with a Wilcoxon-Mann-Whitney test. A logistic procedure with stepwise inclusion of parameters was used to identify key factors that predicted postoperative atrial fibrillation.

\section{Primary Outcome Measure}

The study was designed as a non-inferiority test. Non-inferiority was predefined as a difference of less than 10-mm mean pericardial effusion between the two groups as determined by a calculated 95\% confidence interval around the mean difference in effusions between the groups. The calculation of the nonparametric $95 \%$ confidence interval for effusion used CIA (Confidence Interval Analysis, v2.0.0, T. Bryant, University of Southampton). This statistical analysis was predetermined on the basis of examination of data from a previous study by our group. ${ }^{6}$ This illustrated a predominance of zero effusion in postoperative patients, meaning the data were nonparametric but the median value was invariably zero. Hence median is a poor comparison measure of primary end point and so is mean. Our statistical analysis used a calculated $95 \%$ confidence interval around the mean difference between groups, which, given the distribution of the data, was considered the most appropriate method of comparison.

\section{Results}

Of the 199 patients treated, 109 received Portex drains and 90 received Blake drains. Preoperative or intraoperative variables were well matched (Table 1). Other surgical procedures performed were as follows: isolated single valve replacement in $10.1 \%$ and $13 \%$ in Blake and Portex groups, respectively; combined coronary artery bypass grafting and single valve replacement in $13.5 \%$ and $6.5 \%$, respectively; mitral valve repair in $9 \%$ and $7.4 \%$, respectively; CABG and mitral repair in $2.2 \%$ and $6.5 \%$, respectively; and double valve procedures in $5.5 \%$ and $0.9 \%$, respectively.

If the pleural cavity was breached, drainage depended on the size of the breach and consultant protocol. The left pleura was opened in $83.7 \%$ of the Blake patients and $89 \%$ of the Portex patients, with left pleural drains placed in $44.4 \%$ and $48.6 \%$, respectively. Right pleural breach occurred in $34.7 \%$ (Blake) and $34.3 \%$ (Portex) with right 
TABLE 1. Demographic and operative characteristics of the two patient groups

\begin{tabular}{lccc}
\hline Characteristic & $\begin{array}{c}\text { Blake } \\
(\mathbf{n}=\mathbf{9 0})\end{array}$ & $\begin{array}{c}\text { Portex } \\
(\mathbf{n}=\mathbf{1 0 9})\end{array}$ & $\boldsymbol{P}$ value \\
\hline Female (\%) & 30.0 & 31.2 & .83 \\
Age & $65.7(10.5)$ & $63.6(8.5)$ & .11 \\
Preop creatinine (mmol/L) & $97.5(24.9)$ & $96.9(22.1)$ & .80 \\
Parsonnet score & $10.4(7.7)$ & $8.2(5.8)$ & .021 \\
Normal LV (\%) & 65.6 & 67.9 & .68 \\
CABG only (\%) & 59.6 & 65.7 & .38 \\
Operation time (h) & $3.3(0.9)$ & $3.3(0.8)$ & .74 \\
CPB time (min) & $72(36)$ & $78(36)$ & .79 \\
Crossclamp time (min) & $48(30)$ & $54(30)$ & .62 \\
\hline
\end{tabular}

Results are expressed as percent or mean (standard deviation). $L V$, Left ventricle; $C A B G$, coronary artery bypass grafting; $C P B$, cardiopulmonary bypass.

pleural drains placed in $15.6 \%$ and $18.3 \%$ of patients, respectively.

Despite a trend toward higher maximal hourly drainage rate and total drainage in the Portex group, these differences did not reach statistical significance (Table 2).

There was no significant difference between the size of residual pericardial effusions between the two groups, although a higher percentage of the Blake drain group had an echocardiographically detectable pericardial effusion (Table 2). The $95 \%$ confidence intervals describing the primary outcome measure of difference in effusion between the two groups crosses zero. This illustrates that presence or absence of pericardial effusion is a weak comparative test between drain type, as it does not discriminate between an

TABLE 2. Drain number, duration, and drainage volumes of the two groups

\begin{tabular}{|c|c|c|c|}
\hline Variable & $\begin{array}{c}\text { Blake } \\
(n=90)\end{array}$ & $\begin{array}{l}\text { Portex } \\
(n=109)\end{array}$ & $P$ value \\
\hline \multicolumn{4}{|l|}{ No. of drains per patient } \\
\hline Mean (SD) & $2.1(0.4)$ & $2.0(0.5)$ & NS \\
\hline Median (range) & $2.0(2-3)$ & $2.0(1-3)$ & NS \\
\hline Drain duration (h) & $26.4(16.2)$ & $25.9(11.3)$ & .781 \\
\hline Total drainage $(\mathrm{mL})$ & $541(446)$ & $679(589)$ & .066 \\
\hline $\begin{array}{l}\text { Mean maximal hourly } \\
\text { drainage }(\mathrm{mL})\end{array}$ & $94.7(79.8)$ & $123.1(130.7)$ & .070 \\
\hline $\begin{array}{l}\text { Patients with detectable } \\
\text { effusion }(\%)\end{array}$ & 46.3 & 27.4 & .011 \\
\hline $\begin{array}{l}\text { Mean maximal pericardial } \\
\text { effusion }(\mathrm{mm})\end{array}$ & $5.1(7.2)$ & $3.0(6.3)$ & .053 \\
\hline $\begin{array}{l}\text { Mean Blake-Portex } \\
\qquad(95 \% \text { Cl })\end{array}$ & \multicolumn{3}{|c|}{$1.96 \mathrm{~mm}(-0.02$ to +3.95$)$} \\
\hline
\end{tabular}

The primary outcome statistic is highlighted in bold type. The range is lower than the predefined limit to determine non-inferiority. Data are expressed as mean (standard deviation [SD]) unless otherwise stated. NS, Not significant; $\mathrm{Cl}$, confidence interval.
TABLE 3. Secondary outcome measures of the study population

\begin{tabular}{lccc}
\hline Variable & $\begin{array}{c}\text { Blake } \\
(\mathbf{n}=\mathbf{9 0})\end{array}$ & $\begin{array}{c}\text { Portex } \\
(\mathbf{n}=\mathbf{1 0 9})\end{array}$ & $\boldsymbol{P}$ value \\
\hline Pneumothorax (\%) & 7.8 & 7.4 & .878 \\
Pleural effusion (\%) & 45.6 & 49.1 & .625 \\
Atrial fibrillation (\%) & 48.9 & 38.9 & .154 \\
Respiratory infection (\%) & 15.6 & 11.1 & .382 \\
Resternotomy for & 5.6 & 5.6 & .979 \\
$\quad$ bleeding (\%) & & & \\
Tamponade (\%) & 2.2 & 0.0 & .951 \\
$\begin{array}{l}\text { Death (\%) } \\
\text { Further chest drain }\end{array} \quad 3.3$ & 2.8 & .812 \\
$\quad$ insertion (\%) & 8.8 & 7.2 & $\mathrm{NS}$ \\
Late drainage of & & & \\
$\quad$ pericardial effusion (\%) & 1.1 & 1.9 & $\mathrm{NS}$ \\
\hline
\end{tabular}

No statistical difference is demonstrated between groups. Data are expressed as percent. NS, Not significant.

effusion of $1 \mathrm{~mm}$ and one of $9 \mathrm{~mm}$. This was the reason the primary outcome measure to define equivalence was chosen, and our results indeed demonstrate that the criteria for non-inferiority of the Blake drains are satisfied.

Table 3 describes the adverse events in the study and demonstrates no differences between the two groups. The incidence of pleural effusion is high in both groups, but no distinction was made between tiny, small, and moderate effusions. The majority of effusions noted were tiny in both groups, as reflected by the low incidence of repeat chest drainage procedures required.

As there was a trend toward higher atrial fibrillation rate in the Blake group, we performed multivariate logistic regression analysis to define factors predictive of postoperative atrial fibrillation. The only significant factors identifiable were patient age and operation other than coronary artery bypass graft alone. Neither drain type nor size of effusion was predictive of atrial fibrillation.

\section{Discussion}

Safe, effective drainage of the chest after cardiac surgery is essential to prevent cardiac tamponade, to identify lifethreatening hemorrhage, and to reduce the incidence of symptomatic pleural and pericardial effusions. Despite numerous attempts in cardiac surgery toward smaller incisions and minimally invasive approaches, the chest drainage system has not changed for years. Use of large-bore rigid chest tubes continues at the price of associated discomfort. ${ }^{1,7} \mathrm{~A}$ recent article addressed the issue of pain during drain removal, concluding that analgesia during drain removal is a challenge. ${ }^{8}$ Correspondence published in response suggested that the only way to reduce patient discomfort is to use smaller and softer drains. Blake drains fulfill this criterion and have been available for a number of years. Fears 
over suboptimal performance seem to have prevented their wider use.

Our study illustrates that Blake drains performed in a non-inferior manner to larger Portex drains, with the primary outcome measure being depth of postoperative pericardial effusion. The study set clear limits for the definition of non-inferiority, which were developed on the basis of detecting a performance difference that would change clinical practice. The $95 \%$ confidence interval of the mean difference between groups, our predetermined primary outcome measure, fell well short of the $10-\mathrm{mm}$ non-inferiority limit. It also crossed zero with a maximal value of $3.95 \mathrm{~mm}$, which is too small a difference to affect clinical practice. Hence we clearly demonstrate non-inferiority between the drains.

This study was not designed to detect a difference in reopening for bleeding or tamponade between the treatments. To detect such a difference, a much larger number of patients would have been required. However, our results of equal safety profile agree with other previously published case-control studies of small and large size. In 2000 Obney and associates ${ }^{4}$ reported the use Blake drains for mediastinal drainage after cardiac surgery with no increased risk of tamponade in a case series of 100 patients; they also reported less pain with the smaller drains. Since then, a further retrospective case series has reported adequate performance of Blake drains, ${ }^{5}$ and a retrospective nonrandomized case-control study of 554 patients showed equal efficacy of Blake drains as compared with conventional drains, but with greater patient mobility in the Blake group. ${ }^{9}$

Akowauh and colleagues ${ }^{6}$ performed a prospective randomized controlled trial of Blake drains versus Portex drains in 2002, which demonstrated less pain in the Blake group along with equivalent efficacy, including echocardiography of all patients. This, however, was a study of only 70 patients with 35 in each group.

Recently, Ege and associates ${ }^{10}$ have reported a larger randomized controlled trial investigating the use of Blake drains after cardiac surgery. However, this study randomized patients to standard drains or standard drains plus infracardiac Blake drains and hence the safety of sole Blake drain usage was not evaluated. Interestingly, Ege's group defined an advantage of the addition of an infracardiac Blake drain to the standard drain configuration. We did not identify any advantage of Blake drains over standard Portex drains; however, our study did clearly define the noninferiority of the smaller drains.

In conclusion, this randomized controlled trial confirms that the performance of small Blake drains was similar to that of larger Portex drains after cardiac surgery. This confirms other retrospective reports and smaller randomized controlled trials. As there is evidence that smaller, more flexible drains cause less pain than the larger alternative, on the basis of our results it is justified to use them routinely for most cardiac surgical patients.

\section{Study Limitations}

This study was designed to evaluate two different drain types in the setting of everyday cardiac surgical practice. Limiting the study population to only one type of surgical procedure would make the results difficult to extrapolate to other patient groups. We believe this study achieved a reflection of routine practice. However, by doing so, the study incorporates elements of variability that are difficult to control for. Factors such as variability in the use of bone wax, the use of bilateral internal thoracic arteries, and anticoagulation regimens for tissue valves may all have small effects on the likelihood of detectable postoperative pericardial effusions. However, we suggest that these effects are minor and should not affect a well-defined primary end point.

The other main study limitation is that consultants in charge of patient care made decisions such as when to insert further chest drains when they were not blinded to the drain type the patient received. Again, we would suggest that the primary end point is unlikely to have been affected by consultant knowledge. However, we concede the possible source of bias for secondary end points.

Finally, this study was designed as a test of noninferiority against a predefined standard. Hence it is possible that there is a small performance difference between the two drain types in the region of 1 or $2 \mathrm{~mm}$ of postoperative pericardial effusion that was not detected in this study due to the study size. However, we would suggest that should such a difference exist, it is too small to have any clinical relevance. Hence we are satisfied with our conclusion of non-inferiority of Blake drains.

We thank the echocardiography staff at Glenfield Hospital and Northern General Hospital for their efforts in collecting the echocardiography data for this study.

\section{References}

1. Gift A, Bolgiano C, Cunningham J. Sensations during chest tube removal. Heart Lung. 1991;20:131-7.

2. Chapin J, Kahre J, Newland M. Acute myocardial ischaemia caused by mediastinal chest tube suction. Anesth Analg. 1980;59:386-7.

3. Svedjeholm R, Hakanson E. Postoperative myocardial ischemia caused by chest tube compression of vein graft. Ann Thorac Surg. 1997;64:1806-8.

4. Obney JA, Barnes MJ, Lisagor PG, Cohen DJ. A method for mediastinal drainage after cardiac procedures using small silastic drains. Ann Thorac Surg. 2000;70:1109-10.

5. Lancey RA, Gaca C, Vander Salm TJ. The use of smaller, more flexible chest drains following open heart surgery: an initial evaluation. Chest. 2001;119:19-24.

6. Akowuah E, Ho E, George R, Brennan K, Tennant S, Braidley P, et al. Less pain with flexible fluted silicone chest drains than with conventional rigid chest tubes after cardiac surgery. J Thorac Cardiovasc Surg. 2002;124:1027-8. 
7. Paiement B, Boulanger M, Jones C, Roy M. Intubation and other experiences in cardiac surgery: the consumers view. Can Anaesth Soc J. 1979;26:173-80.

8. Barnard J, Thompson J, Dunning J. Can any intervention effectively reduce the pain associated with chest drain removal? Interactive Cardiovasc Thorac Surg. 2004;3:229-32.
9. Frankel TL, Hill PC, Stamou SC, Lowery RC, Pfister AJ, Jain A, et al. Silastic drains vs conventional chest tubes after coronary artery bypass. Chest. 2003;124:108-13.

10. Ege T, Tatli E, Canbaz S, Cikirikcioglu M, Sunar H, Ozalp B, et al. The importance of intrapericardial drain selection in cardiac surgery. Chest. 2004;126:1559-62.

\section{Interactive eLearning Activities \\ http://learning.ctsnet.org}

The Joint Council on Thoracic Surgery Education was pleased to introduce a series of unique eLearning activities to CTSNet users at the AATS annual meeting in Philadelphia. Sponsored by several cardiothoracic surgical groups, this exciting new educational tool contains narrated videos of actual surgical procedures followed by a series of questions and an evaluation to earn Continuing Medical Education credit.

* Diagnosis and Management of Complications of Mitral Valve Repair

* Off Pump Coronary Artery Bypass

* OPCAB Debriefing

* Pediatric Cardiopulmonary Bypass Emergency Situations

Experience these new educational tools today and provide us with your feedback. Go to: http://learning.ctsnet.org

William E. Baumgartner, Chair

Joint Council on Thoracic Surgery Education

Sponsored By:

American Association for Thoracic Surgery

The Society of Thoracic Surgeons

European Association for Cardio-Thoracic Surgery

Children's Memorial Hospital 